BULLETIN Bulletin hispanique

HispaniQuE Université Michel de Montaigne Bordeaux

$116-2$ | 2014

Référentialité/autoréférentialité dans le roman

espagnol contemporain : bilan et perspectives

\title{
Jaime Siles, Sémaphores, sémaphores
}

Presses Universitaires Blaise Pascal, Clermont-Ferrand, 2013

\section{Monique Plâa}

\section{(2) OpenEdition}

\section{Journals}

Édition électronique

URL : http://journals.openedition.org/bulletinhispanique/3684

DOI : 10.4000/bulletinhispanique.3684

ISBN : 979-10-300-0156-3

ISSN : $1775-3821$

Éditeur

Presses universitaires de Bordeaux

\section{Édition imprimée}

Date de publication : 1 décembre 2014

Pagination : 932-934

ISBN : 978-2-86781-963-6

ISSN : 0007-4640

Référence électronique

Monique Plâa, « Jaime Siles, Sémaphores, sémaphores », Bulletin hispanique [En ligne], 116-2 | 2014, mis en ligne le 01 décembre 2017, consulté le 23 septembre 2020. URL : http://journals.openedition.org/ bulletinhispanique/3684; DOI : https://doi.org/10.4000/bulletinhispanique.3684

Ce document a été généré automatiquement le 23 septembre 2020.

Tous droits réservés 


\title{
Jaime Siles, Sémaphores, sémaphores
}

Presses Universitaires Blaise Pascal, Clermont-Ferrand, 2013

\author{
Monique Plâa
}

\section{RÉFÉRENCE}

Jaime Siles, Sémaphores, sémaphores. Clermont-Ferrand, Presses Universitaires Blaise Pascal, 2013, 208 p. (collection Celis Textes). Traduit par Henry Gil. - ISBN-13:

9782845166196

1 En 1999, Jaime Siles [né en 1951] publie Himnos tardíos, son livre le plus connu, un recueil qu'il dit triste mais auquel il avoue être particulièrement attaché. Himnos tardíos est composé de 5 sections, "El Oro de los días », « Himnos tardíos ", « Partida de ajedrez », " Vita philologica» et «Ángulos muertos» qu'accompagne une liste de dédicaces dont la première dit : «El oro de los días a su mejor intérprete: al hispanista Henry Gil. »

2 En 2003, Henry Gil traduit Hymnes tardifs pour les éditions Circé. À la fin de l'ouvrage figure un texte, Le spectacle de l'être, où, en interprète subtil et profondément attentif à l'écriture de Jaime Siles, Henry Gil permet au lecteur d'appréhender l'œuvre du poète " novísimo» dans ses étapes successives. Quasi abstrait, d'une grande économie de moyens, auteur de recueils au bord de la métapoétique et au bord du silence dans ses débuts, Jaime Siles, qui écrit peu et méticuleusement, donne à lire une œuvre en évolution lente mais nette. Après l'étape d'un Moi «grandi à l'ombre des dictionnaires » et jonglant avec la langue pour en privilégier le signe au point de prôner la dé-signification, vient un Moi davantage incarné, moins essentiel et plus contingent, plus concret et plus présent qui, dans Hymnes tardifs, s'inscrit douloureusement dans la perception lucide de la perte dont seule l'écriture pourrait éventuellement exorciser la mélancolique inéluctabilité.

3 Entre ces deux étapes, il y a, publié en 1989, Sémaphores, Sémaphores, un recueil de transition qui s'éloigne de la première époque, celle du poème-instant des abstractions et de la dé-signification conceptiste, et annonce l'époque qui suivra, celle d'un Moi élégiaque pris et emporté dans le mouvement incessant du Temps et inscrit dans des 
poèmes-discours comparativement beaucoup plus longs que ceux de la première époque. Pourtant Sémaphores, sémaphores a aussi une identité propre : Siles s'éloigne de l'abstraction mais n'est pas encore envahi par l'omniprésence d'un Temps oppressant et destructeur et, dans cette parenthèse, Sémaphores, sémaphores s'ouvre discrètement au monde et à ses scintillements.

C'est ce recueil, primé comme tant de recueils de Jaime Siles mais, injustement, un peu moins commenté et un peu moins cité que d'autres, que les éditions des Presses Universitaires Blaise Pascal ont eu, en 2013, la très bonne idée de publier dans une traduction d'Henry Gil qui, pour la circonstance, met à nouveau ses talents d'interprète au service de cette forme particulière d'interprétation qu'est la traduction. Le livre comporte, outre une introduction du traducteur - «Sémaphores, sémaphores ou les prémices d'un lyrisme existentiel »- un avant-propos, également du traducteur, où le lecteur découvrira la rigueur extrême que requiert, sous des apparences humbles, l'ambitieux projet « d'obtenir avec des moyens différents des effets analogues ».

5 Henry Gil traduit les vers en vers. Ce choix de traduction, le plus exigeant mais sans doute le seul approprié lorsque le texte source jongle constamment avec les mètres et les rimes, les sons et les rythmes, suppose de suivre la règle de la TraductionRecréation où « tout l'art du traducteur consiste précisément à ne pas faire de sacrifices au delà du nécessaire, à ne tolérer les transformations que si elles demeurent dans le cadre précis et restreint du système artistique en question, à ne faire d'additions que si elles ne franchissent pas les bornes du monde esthétique du poète ».

Dès le titre, Sémaphores, sémaphores joue avec la pluralité des sens - « sémaphores » c'est le signalement lumineux et l'invitation au passage mais aussi le porteur de sèmes, $\hat{o}$ combien pluriels! - et, joue, parallèlement, avec le son dont le redoublement semble clignoter pour inviter chacun de nous à la lecture des 26 poèmes du recueil regroupés en trois sections : «Madrigaux urbains", «Elégie et analyse» et "Corps diplomatique ».

7 On assiste, dans Sémaphores, sémaphores, à l'apparition d'un Moi aux occurrences multiples et incertaines, toutes pareillement inscrites dans le mouvement du Temps qui insidieusement précipite l'Être dans le non-Être, ce qui n'empêche pas le locuteur de s'amuser parfois d'un certain Jaime - Jaime, vieux camarade, je te dis :/ nous sommes somas car nous sommes signes/; ni parfois de se moquer d'un innocent Jaime Siles, - Jaime Siles, ce poète très froid,/ si partisan de toute suppression -, dont l'écriture première manière dit bien le temps pensé et conceptualisé mais mal le temps ressenti et constaté qui hante désormais le poète - "[...] en ressentant sur ses yeux en vermeil/ que le temps est plus que simple fiction/ créée par les mots [...] ». C'est ainsi que ce locuteur, si près du poète qu'il lui arrive de porter son nom et son prénom, se retrouve en train de philosopher devant une boîte d'allumettes - [...] «sur cette simple boite d'allumettes/ afin d'écrire son temps s'écrivit» - offrant au lecteur un amusement qui fait efficacement contrepoint à l'assombrissement qui menace. C'est que la note élégiaque, qui emportera le locuteur et prendra un tour nettement mélancolique dans Hymnes tardifs, est ici contrebalancée par l'éclat que donne au texte l'attention portée aux choses et au monde, aux villes et aux femmes, aux peintures et aux corps, aux tableaux et aux poèmes.

8 En 87, Jaime Siles, alors Directeur de l'Institut culturel espagnol à Vienne, publie Viena, un joli livre sur la capitale autrichienne dont les titres de chapitre, sophistiqués et amusants par le jeu de références et l'agencement délibérément chaotique - « I. Introito lírico, memorial de consejos prácticos, dónde cenar la primera noche y cómo 
terminarla en un café »-, semblent annoncer le tour et le ton imprimé à nombre de poèmes de Sémaphores, sémaphores et tout particulièrement à ceux de la section "Madrigaux urbains». L'urbain, dans Sémaphores, sémaphores, c'est le lieu des tentations: des blondes incendiaires surgissent ici ou là, flamboyantes et fardées, apparemment toujours offertes mais finalement malaisées à conquérir et la reddition, quand elle a lieu, prend des airs épico-comiques: L'aube à la pointe de ses traits donna/ une teinte de tulipes fuchsia./ Les cormorans à l'adresse aérienne// luttèrent au soleil une semaine/ et tombèrent en vaillants capitaines/ morts en défendant ses cônes grenat. Cette nuance d'amusement, cette tentation de la légèreté, qui n'exclut pas la pointe élégiaque mais lui tient encore la bride haute, donne toute sa saveur à ce recueil pris entre deux esthétiques plus graves et, pour celle qui viendra après, singulièrement plus mélancolique.

Dans le poème intitulé "Ulysse et les sirènes ", assorti d'une parenthèse qui nous avertit qu'il s'agit d'une version "libre et modernisée », le locuteur, attiré dans un bar par une sirène en topless, finit une nuit très alcoolisée dans un état très incertain : Arrivant ainsi à un endroit qui,/par son profil, rappelait le matin/ je me suis donc enquis du jour enfui// afin de trouver la semaine enfin./ Ni tram ni métro ne venant au loin/ c'est dans un autre club que j'ai fini. Dans l'ensemble du recueil, le locuteur est un héros très aléatoire lancé dans des périples qui ne le sont pas moins : le monde bouge et le Moi avec, le monde varie et le Moi aussi ; tout tend à se répéter mais dans le changement, ce qu'on a vu est soudain escamoté, ce qui revient n'est plus pareil et le monde devient un kaléidoscope dont le poème capte les miroitements baroques. Le poème le plus sophistiqué et le plus long du recueil, «Corps diplomatique » - qui constitue à lui seul la totalité de la troisième section -, est un constant glissement d'une chose vers une autre: la ville, la femme et la peinture sont autant de motifs qui tournoient et virevoltent sans fin au fil des hendécasyllabes devenus alexandrins en français. Et quoique le Temps, comme toujours dans le monde de Siles, soit une menace, "Corps diplomatique » est d'abord une fête, une célébration dont le poème témoigne dans une dynamique comme ivre de ses variations.

Dans « Corps diplomatique », la fête doit certes beaucoup à la femme désirée et chantée mais aussi à la peinture sans laquelle rien ne serait: L'or de leur corps, le tableau de Gauguin, est un motif essentiel soumis à variation du début à la fin du poème. De la plus immédiate à la plus gongorine, Sémaphores, sémaphores recourt constamment à la métaphore qui dit la sensation et la perception où cristallisent mémoire et imagination. Dans le registre métaphorique, le champ du visuel est particulièrement privilégié. Le poète convoque l'iconographie publicitaire, l'esthétique du vidéo-clip et du cinéma, les compositions cubistes, fauves ou impressionnistes, mais également le lyrisme abstrait et, d'une manière générale le dessin et surtout les couleurs dans leur infinie diversité chromatique. Mallarmé et Manet, Max Jacob et Picasso, Leiris et Miro, André du Bouchet et Antoni Tapies, parmi tant d'autres, ont fait dialoguer la poésie et l'image par le livre. Sémaphores sémaphores se situe dans un au-delà du dialogue : du cœur même des poèmes jaillissent des images qui créent un musée imaginaire pour un lecteur constamment invité à partager la fête subtile et toujours mouvante des formes et des couleurs. 


\section{AUTEURS}

\section{MONIQUE PLÂA}

Université Paris-Est Marne-la-Vallée 